\title{
Ceria nanoclusters on graphene/Ru(0001): A new model catalyst system
}

\author{
Z. Novotny ${ }^{\dagger}$, F.P. Netzer ${ }^{* *}$, Z. Dohnálek $^{+*}$
}

${ }^{\dagger}$ Physical and Computational Sciences Directorate and Institute for Interfacial Catalysis, Pacific Northwest National Laboratory, Richland, Washington 99354, United States

${ }^{\ddagger}$ Surface and Interface Physics, Institute of Physics, Karl-Franzens University, A-8010 Graz, Austria

\section{Corresponding Authors}

*Corresponding authors: falko.netzer@uni-graz.at and zdenek.dohnalek@pnnl.gov 


\section{Abstract}

The growth of ceria nanoclusters on single-layer graphene on $\mathrm{Ru}(0001)$ has been examined, with a view towards fabricating a stable system for model catalysis studies. The surface morphology and cluster distribution as a function of oxide coverage and substrate temperature has been monitored by scanning tunneling microscopy (STM), whereas the chemical composition of the cluster deposits has been determined by Auger electron spectroscopy (AES). The ceria nanoparticles are of the $\mathrm{CeO}_{2}(111)$-type and are anchored at the intrinsic defects of the graphene surface, resulting in a variation of the cluster densities across the macroscopic sample surface. The ceria clusters on graphene display a remarkable stability against reduction in ultrahigh vacuum up to $900 \mathrm{~K}$, but some sintering of clusters is observed for temperatures $>450 \mathrm{~K}$. The evolution of the cluster size distribution suggests that the sintering proceeds via a Smoluchowski ripening mechanism, i.e. diffusion and aggregation of entire clusters. 


\section{Keywords}

Ceria, $\mathrm{CeO}_{2}$, nanoclusters, graphene, $\mathrm{Ru}(0001)$, Smoluchowski ripening, STM, AES

\section{Graphical Abstract}

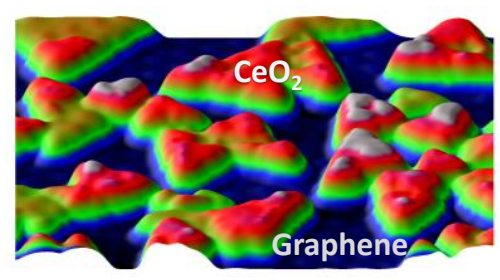

\section{Introduction}

The cerium atom with its $4 f^{1}(5 d 6 s)^{3}$ outer electron configuration supports two stable oxide stoichiometries, $\mathrm{Ce}_{2} \mathrm{O}_{3}$ and $\mathrm{CeO}_{2}$, containing $\mathrm{Ce}^{3+}\left(4 \mathrm{f}^{1}\right)$ and $\mathrm{Ce}^{4+}\left(4 \mathrm{f}^{0}\right)$ cationic species, respectively. The energetic near-degeneracy of the core-like $4 f^{1}$ electron and the extended $(5 \mathrm{~d} 6 \mathrm{~s})^{3}$ valence states enables an easy electron transfer between these states and thus between the $3+$ and $4+$ oxidation states of cerium cations. This is at the root of the flexible reduction-oxidation behavior of cerium oxides, which forms the basis of their widespread interest in science and technology. The transformation of $\mathrm{Ce}^{4+}$ to $\mathrm{Ce}^{3+}$ and vice versa is typically associated with the creation/annihilation of oxygen vacancies, and the ability to store or release oxygen in response to the chemical environment is the one outstanding feature of cerium oxides. Cerium oxides are used in many different areas of catalysis $[1,2]$, 
in solid oxide fuel cells [3], and applications are discussed even in pharmacology due to their antioxidant properties [4]. Cerium oxides are contained as catalysts in the three-way converter for automotive exhaust control [5], they are active in the water-gas shift reaction $[6,7]$, in the steam reforming of oxygenates [8], in soot oxidation [9], in the preferential oxidation of $\mathrm{CO}[10]$ and in the activation of $\mathrm{CO}_{2}$ [11]. It has been recognized in the last two decades that thin films of oxides supported on metal surfaces provide a very useful concept as model systems for catalysis studies, allowing the controlled investigation of elementary reaction steps at the molecular level [12]. This approach has been also extensively employed for cerium oxides, and thin films of cerium oxides, with a view towards model catalyst, have been grown on $\mathrm{Ru}(0001)$ [13, 14], $\mathrm{Rh}(111)$ [15-18], $\mathrm{Pd}(111)$ [19], $\mathrm{Ni}(111)$ [13], $\mathrm{Cu}(111)$ [2022], $\operatorname{Re}(0001)$ [23], $\mathrm{Pt}(111)$ [24-26], and flat and stepped $\operatorname{Au}(111)[27,28]$ surfaces. The primary target oxide of thin film growth in the majority of these studies was $\mathrm{CeO}_{2}$ (ceria), to provide a well-defined starting stoichiometry for subsequent catalytic reaction studies.

The metal supported ceria thin films mentioned above provide suitable planar model systems for catalysis, however is has been recognized recently that ceria in systems with nanoscale dimensions exhibits significantly enhanced catalytic activity as compared to systems at larger size scales and morphologies $[7,9,11,29,30]$. The support material of ultrathin ceria films or nanostructures has an influence on the stability of the oxide phases, both in terms of oxidation state and morphology. Metal surfaces are convenient substrates for oxide nanostructures concerning surface preparation and characterization with typical surface science techniques [31], but as electron donors they tend to facilitate the reduction of ceria in ultrathin films or nanostructures $[15,16,22,26,32]$. Also, the sintering of oxide nanoparticles during annealing and eventually under chemical reaction conditions has to be considered for the practicality of the structures as catalytic model systems. Support surfaces 
that are chemically inert and provide stable anchoring conditions for nanoparticles are desirable for a successful catalyst model system. Here, we propose single layer graphene on $\mathrm{Ru}(0001)$ as a suitable substrate for ceria nanocluster growth.

We have investigated graphene $(\mathrm{Gr})$ on $\mathrm{Ru}(0001)$ as a support surface for the growth and stabilization of ceria nanoparticles using scanning tunneling microscopy (STM) to trace the morphology and thermal stability of ceria nanoclusters and to evaluate the evolution of cluster density and size distribution as a function of the ceria coverage, the growth conditions and thermal annealing. The stoichiometry of the ceria clusters has been evaluated using Auger electron spectroscopy (AES). We find that the intrinsic defects in the graphene layer act as anchoring centers for the ceria nanoclusters and that the ceria cluster array is robust against thermal reduction up to temperatures beyond $900 \mathrm{~K}$ in ultra-high vacuum (UHV). Moderate sintering of the ceria nanoparticles sets in at $\mathrm{T}=400-500 \mathrm{~K}$ and there are indications of the Smoluchowski ripening mechanism, i.e. cluster growth via cluster migration and agglomeration. It should also be noted that the ceria nanoclusters, in the presence of oxygen, are active in promoting the intercalation of oxygen between the graphene layer and the Ru surface at moderate temperatures (600 K) via an oxygen spillover effect, as reported recently [33].

\section{Experimental}

All experiments have been carried out in a UHV system with a base pressure below $1 \times 10^{-10}$ Torr. STM images were obtained using a commercial variable-temperature STM (Omicron) operated at room temperature $(300 \mathrm{~K})$ in constant current mode and using electrochemically etched W tips. Acquired images were processed by ImageJ software [34]. The processing 
included background subtraction and noise removal from frequency domain images. Distortion of the STM images was removed using algorithms described in Ref. [35]. Total number of clusters was determined by analyzing the images using Multi-Point tool contained in the ImageJ software package. The error bars were determined by averaging the counts from at least five different $250 \times 250 \mathrm{~nm}^{2}$ areas on the sample. Cluster height was analyzed using Find Maxima tool for small coverages, where the clusters were isolated and adopted circular 3D shape. For higher coverages, height line profiles were used.

The $\mathrm{Ru}(0001)$ sample (Princeton Scientific) was mounted on a Ta sample plate using a thin Ta foil, and heated using a pyrolytic boron nitride (PBN) heater on the manipulator, or in a dedicated electron-beam annealing stage. The $\mathrm{Ru}(0001)$ temperature in the e-beam annealing stage was measured with an optical pyrometer. On the PBN heating stage, temperature was measured using a thermocouple (type K) spot-welded on the manipulator in a close proximity to the sample. Clean $\mathrm{Ru}(0001)$ was prepared by repeated cycles of $\mathrm{Ne}^{+}$ ion sputtering at $300 \mathrm{~K}$, oxidation in $1 \times 10^{-7}$ Torr $\mathrm{O}_{2}$ at $850 \mathrm{~K}$ and flash-annealing in UHV above $1600 \mathrm{~K}$. A clean surface was verified with low-energy electron diffraction (LEED), AES, and STM.

Gr was grown by chemical vapor deposition of ethylene $\left(\mathrm{C}_{2} \mathrm{H}_{4}\right)$ at $1100 \mathrm{~K}$ for 30 minutes using a custom built tube doser with a $3.07 \mu \mathrm{m}$ pinhole and 8 Torr backing pressure. As grown $\mathrm{Gr} / \mathrm{Ru}(0001)$ exhibits defects (highlighted with white arrows in Fig. 1) surrounded with defect-free Gr Moiré areas (areas encompassed by dotted lines in Fig. 1). These defects are attributed to point defects [36] and domain boundaries between differently oriented $\mathrm{Gr}$ domains, and show a large spatial variation, as demonstrated previously [33]. 


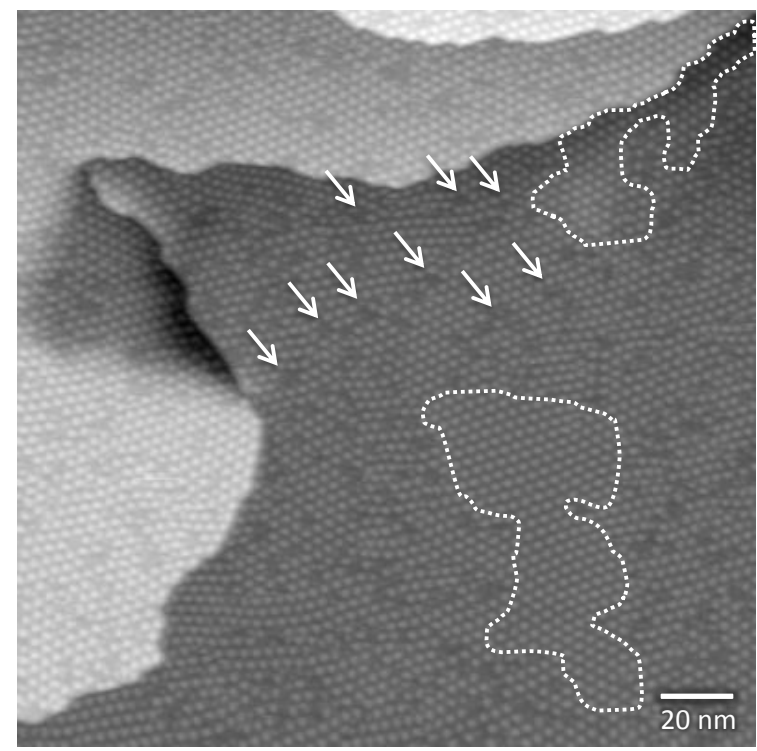

Fig. 1: STM image of single-layer $\mathrm{Gr} / \mathrm{Ru}(0001)$ with a high local density of defects. Defect-free regions with unperturbed Gr Moiré are encompassed with dotted white lines. Several defective regions in the Gr Moiré are highlighted with white arrows. Imaging conditions: $200 \times 200 \mathrm{~nm}^{2}, \mathrm{~V}_{\mathrm{s}}=+0.8 \mathrm{~V}, \mathrm{l}_{\mathrm{t}}=25 \mathrm{pA}$.

Ce (Alfa Aesar, 99.9\%) was deposited from a Ta crucible in a high temperature effusion-cell (CreaTec). The deposition rate was calibrated with a water-cooled quartz crystal microbalance (Inficon) by deposition of $\mathrm{Ce}$ in UHV. $1 \mathrm{ML}$ of $\mathrm{CeO}_{2}$ is defined with respect to the density of the $\mathrm{Ce}$ atoms in the $\mathrm{CeO}_{2}(111)$ surface, $7.88 \times 10^{14} \mathrm{Ce}$ atoms $/ \mathrm{cm}^{2}$, assuming that during reactive deposition of $\mathrm{Ce}$ in $\mathrm{O}_{2}$ background, the evaporation rate of $\mathrm{Ce}$ remains unchanged. We note that the amount of deposited Ce atoms in reference [33] should be multiplied by a factor of 0.6 to obtain the coverage in ML units used in this manuscript [37]. Oxygen was dosed via back filling the UHV chamber using a high-precision leak valve, and pressures were measured with a Bayard-Alpert ion gauge. A fixed value of oxygen pressure of $1 \times 10^{-7}$ Torr and a constant deposition rate of $1.7 \times 10^{-3} \mathrm{ML} / \mathrm{sec}$ was used in all experiments shown in this manuscript. 
The stoichiometry of the ceria clusters was determined by using $12 \mathrm{ML}$ thick ceria films on $\mathrm{Ru}(0001)$. The thick film deposited at $700 \mathrm{~K}$ in $5 \times 10^{-7}$ Torr $\mathrm{O}_{2}$ with the same deposition rate as used in Ref. [13] was taken as a reference of stoichiometric $\mathrm{CeO}_{2}$. AES measurements showed only Ce and O peaks with no Ru peak at this film thickness (data not shown). Vacuum annealing of this film up to $1100 \mathrm{~K}$ showed no detectable change in the oxygen to cerium signal ratio, in agreement with previous studies [13]. Growing another $12 \mathrm{ML}$ thick $\mathrm{CeO}_{x}$ film with the growth conditions described in this manuscript $\left(300 \mathrm{~K}, 1 \times 10^{-7}\right.$ Torr $\mathrm{O}_{2}$ ) showed, within the accuracy of AES (2-3\%), the same ratio of $\mathrm{O}\left(\mathrm{KL}_{23} \mathrm{~L}_{23}\right) / \mathrm{Ce}\left(\mathrm{M}_{45} \mathrm{~N}_{45} \mathrm{~N}_{45}\right)$ as the stoichiometric $\mathrm{CeO}_{2}$ thick film. The same ratio of $\mathrm{O}\left(\mathrm{KL}_{23} \mathrm{~L}_{23}\right) / \mathrm{Ce}\left(\mathrm{M}_{45} \mathrm{~N}_{45} \mathrm{~N}_{45}\right)$ as for stoichiometric $\mathrm{CeO}_{2}$ was then measured for the low ceria coverage of $2.4 \mathrm{ML}$ grown at $300 \mathrm{~K}$ in $1 \times 10^{-7}$ Torr $\mathrm{O}_{2}$ on $\mathrm{Gr} / \mathrm{Ru}(0001)$. The stoichiometry of ceria nanoclusters, i.e. close to $\mathrm{CeO}_{2}$, was further confirmed by weight measurements using the quartz crystal microbalance via the observation of the change in the deposition rate upon introduction of oxygen [38] (the evaporation rate increased by a factor of $\sim 1.26$ between deposition of metallic Ce in UHV and deposition in $1 \times 10^{-7}$ Torr $\mathrm{O}_{2}$ ). Therefore, we conclude that the $\mathrm{CeO}_{\mathrm{x}}$ nanoclusters presented in this manuscript should be near-stoichiometric $\mathrm{CeO}_{2}$.

Because the $\mathrm{Ce}\left(\mathrm{M}_{45} \mathrm{~N}_{45} \mathrm{~N}_{45}\right)$ peak at $661 \mathrm{eV}$ is below the detection limit for $\mathrm{CeO}_{x}$ coverages smaller than $1 \mathrm{ML}$ of $\mathrm{CeO}_{x}$, the $\mathrm{Ce}\left(\mathrm{N}_{45} \mathrm{O}_{23} \mathrm{~N}_{67} / \mathrm{V}\right)$ peak at $82 \mathrm{eV}$ [39] was used to investigate the thermal stability of $\mathrm{CeO}_{x}$ nanoclusters at a fixed initial coverage. The coverage of $\mathrm{Gr}$ in AES spectra was estimated from the ratio of the negative to positive excursions of the 272$273 \mathrm{eV}$ derivative AES peak (representing the overlapping C KVV and $\mathrm{Ru} \mathrm{M}_{45} \mathrm{VV}$ transitions), where the asymmetry of the graphitic carbon signal in AES was utilized, as compared to the relatively symmetric $\mathrm{Ru} \mathrm{M}_{45} \mathrm{VV}$ peak $[40,41]$. 


\section{Results and discussion}

\subsection{Ceria cluster growth at $300 \mathrm{~K}$}
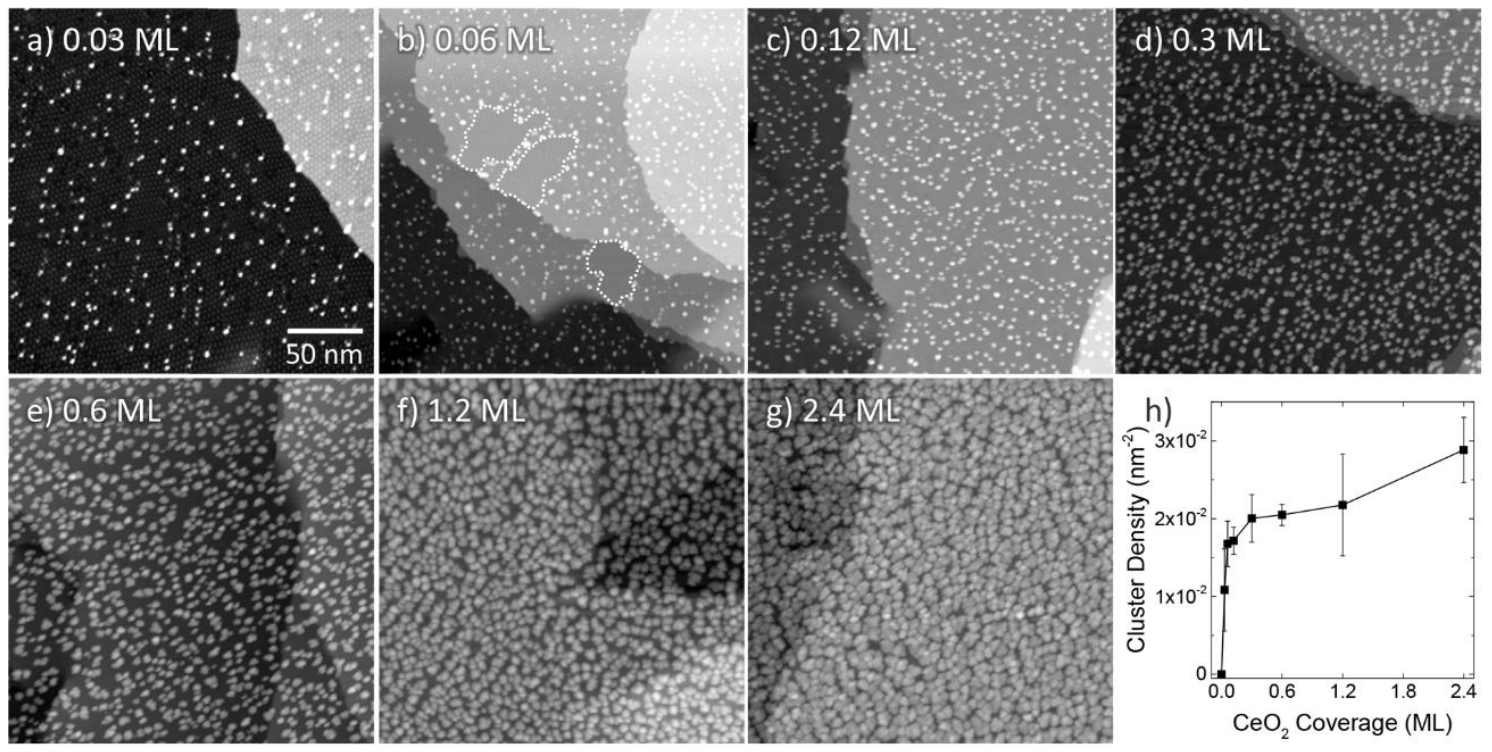

Fig. 2: Ceria nanoclusters deposited on $\mathrm{Gr}$ on $\mathrm{Ru}(0001)$ via reactive deposition of $\mathrm{Ce}$ in $1 \times 10^{-7}$ Torr of $\mathrm{O}_{2}$ at $300 \mathrm{~K}$. Panel (a-g) shows STM images of $\mathrm{CeO}_{\mathrm{x}}$ nanoclusters with coverage increasing from 0.03 ML to 2.4 ML. Defect-free Gr Moiré areas with no $\mathrm{CeO}_{\mathrm{x}}$ clusters present are regularly observed for low $\mathrm{CeO}_{\mathrm{x}}$ coverages, as highlighted by dotted white lines in panel (b). Panel (h) shows the total density of clusters, with error bars estimated from at least five different areas on the sample. Imaging conditions: $250 \times 250 \mathrm{~nm}^{2}, \mathrm{~V}_{\mathrm{s}}=-(1.5-1.6) \mathrm{V}, \mathrm{I}_{\mathrm{t}}=100 \mathrm{pA}$

The overall growth morphology of ceria nanoclusters on graphene/Ru(0001), deposited at $300 \mathrm{~K}$, is illustrated for increasing ceria coverages in the large scale STM images of Fig. 2(a-g). The images displayed here are prototypical, in the sense that they show average cluster densities, but the densities vary to a certain extent across the macroscopic surface. The densities of clusters (cluster counts per $\mathrm{nm}^{2}$ ) as a function of oxide surface coverage are plotted in Fig. 2(h), where the error bars reflect the density variations across the 
macroscopic surface. The local distribution of clusters on the surface is also not completely uniform, there are more crowded regions and there are denuded areas - see, e.g. in Fig. 2(b), where areas bare of any clusters are highlighted by the white dotted lines. In order to further demonstrate spatial variation of the cluster densities across the graphene surface, Fig. 3 shows STM images of $1.2 \mathrm{ML}$ ceria recorded from different sample areas. Image (a) is from a prototypical region with an average cluster density $\left(2.1 \times 10^{-2}\right.$ clusters $\left./ \mathrm{nm}^{2}\right)$, image (b) is from a low-density area $\left(0.7 \times 10^{-2}\right.$ clusters $\left./ \mathrm{nm}^{2}\right)$, whereas image (c) is from a highdensity region $\left(2.6 \times 10^{-2}\right.$ clusters $\left./ \mathrm{nm}^{2}\right)$. In the low-density region of Fig. $3(\mathrm{~b})$, the ceria islands display fractal-like shapes and are larger than average, with typical length dimensions of $\sim 15 \mathrm{~nm}$; in the more densely populated areas (Fig. 3(a,c)), a typical cluster size is $\sim 3-6 \mathrm{~nm}$. Close inspection of the zoom-in image of the low-density region of Fig. 3(b) (inset) reveals that in the space between the clusters the graphene Moiré is unperturbed and shows no defects. No clear preference of the nucleation of the clusters at the step edges is observed in STM images.
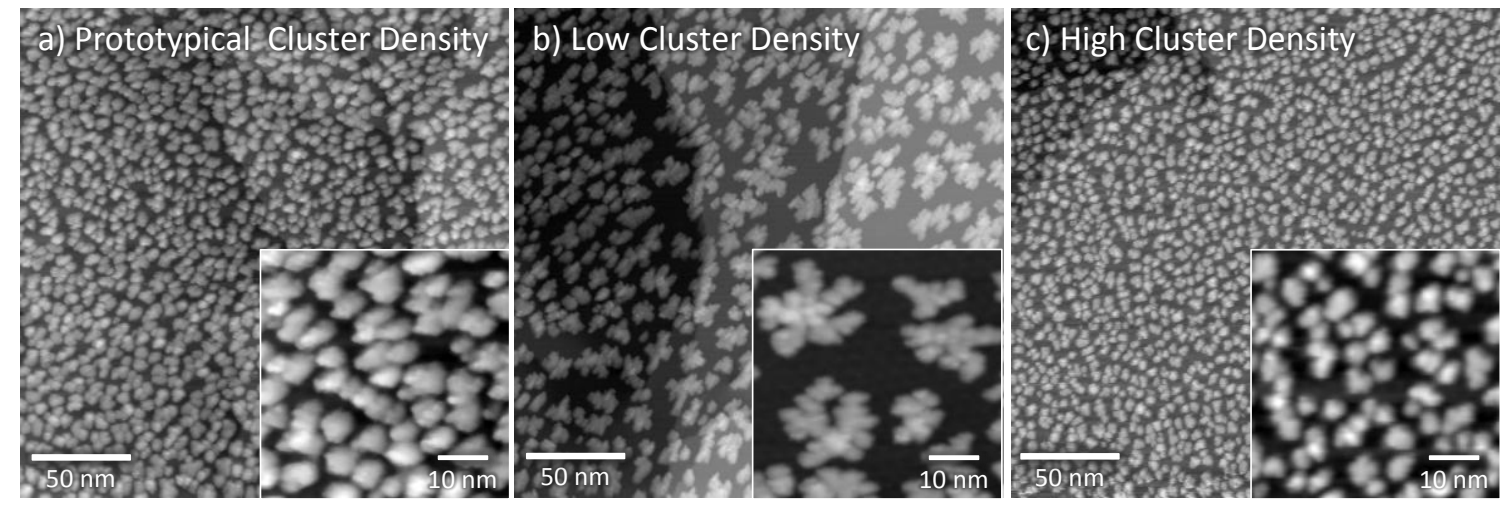

Fig. 3: STM images demonstrating spatial variation of the density of $\mathrm{CeO}_{\mathrm{x}}$ clusters at different areas of the sample within one experiment (1.2 ML Ce deposited in $1 \times 10^{-7}$ Torr of $\mathrm{O}_{2}$ at $300 \mathrm{~K}$ ). Panels (a) show a prototypical image with cluster density corresponding to average cluster density shown in Fig. 2(h). Panel (b) shows an area 
with an extremely low density of $\mathrm{CeO}_{x}$ clusters $(67 \%$ lower than the average density), where $\mathrm{CeO}_{\mathrm{x}}$ forms fractal-like aggregates on the surface, and dark areas representing defect-free Gr Moiré. Panel (c) shows an area with a high density of clusters ( $26 \%$ more than the average density). The magnified images in the insets provide a more detailed view of the clusters. Imaging conditions: $250 \times 250 \mathrm{~nm}^{2}$ (insets $\left.50 \times 50 \mathrm{~nm}^{2}\right), \mathrm{V}_{\mathrm{s}}=-1.6 \mathrm{~V}, \mathrm{I}_{\mathrm{t}}=100 \mathrm{pA}$.

This non-uniform distribution of clusters points towards a defect-mediated growth mechanism. The clusters nucleate at the intrinsic defects of the graphene Moiré lattice [36], formed during the preparation of graphene by high-temperature chemical vapor deposition. The irregular distribution of defects over the bare Gr surface, containing also large defectfree areas, is shown in Fig. 1. Correlation analysis of cluster-cluster separation distances indicates that the clusters are randomly distributed, as are the defects, and this again corroborates the proposed defect based nucleation mechanism. No clear preference for Moiré sites is observed in case of $\mathrm{CeO}_{\mathrm{x}}$ clusters, in contrast to metals deposited on $\mathrm{Gr} / \mathrm{Ru}(0001)$ or $\mathrm{Gr}$ Moiré $[42,43]$. The clusters increase in number and size with increasing ceria coverage as visually clearly apparent in the STM images of Fig. 2. At 2.4 ML ceria (Fig. 2(g)), the clusters start to coalesce. The quantitative analysis of the cluster density in Fig. 2(h) exhibits a non-linear behavior, with a steep increase at low coverages and a change of gradient at $\sim 0.1 \mathrm{ML}$, followed by a slower increase. This behavior suggests that nucleation sites of different cluster binding strength exist on the graphene surface. At low ceria coverage, the strong binding sites trap the incoming cerium adatoms leading to a high nucleation density and to a rapid increase of the number of clusters. After saturation of these primary nucleation sites, at $\sim 0.1 \mathrm{ML}\left(\sim 2 \times 10^{-2}\right.$ clusters $\left./ \mathrm{nm}^{2}\right)$ in Fig. 2(h), weaker 
bonding sites lead to secondary nucleation processes and concomitantly to an increase in cluster size.
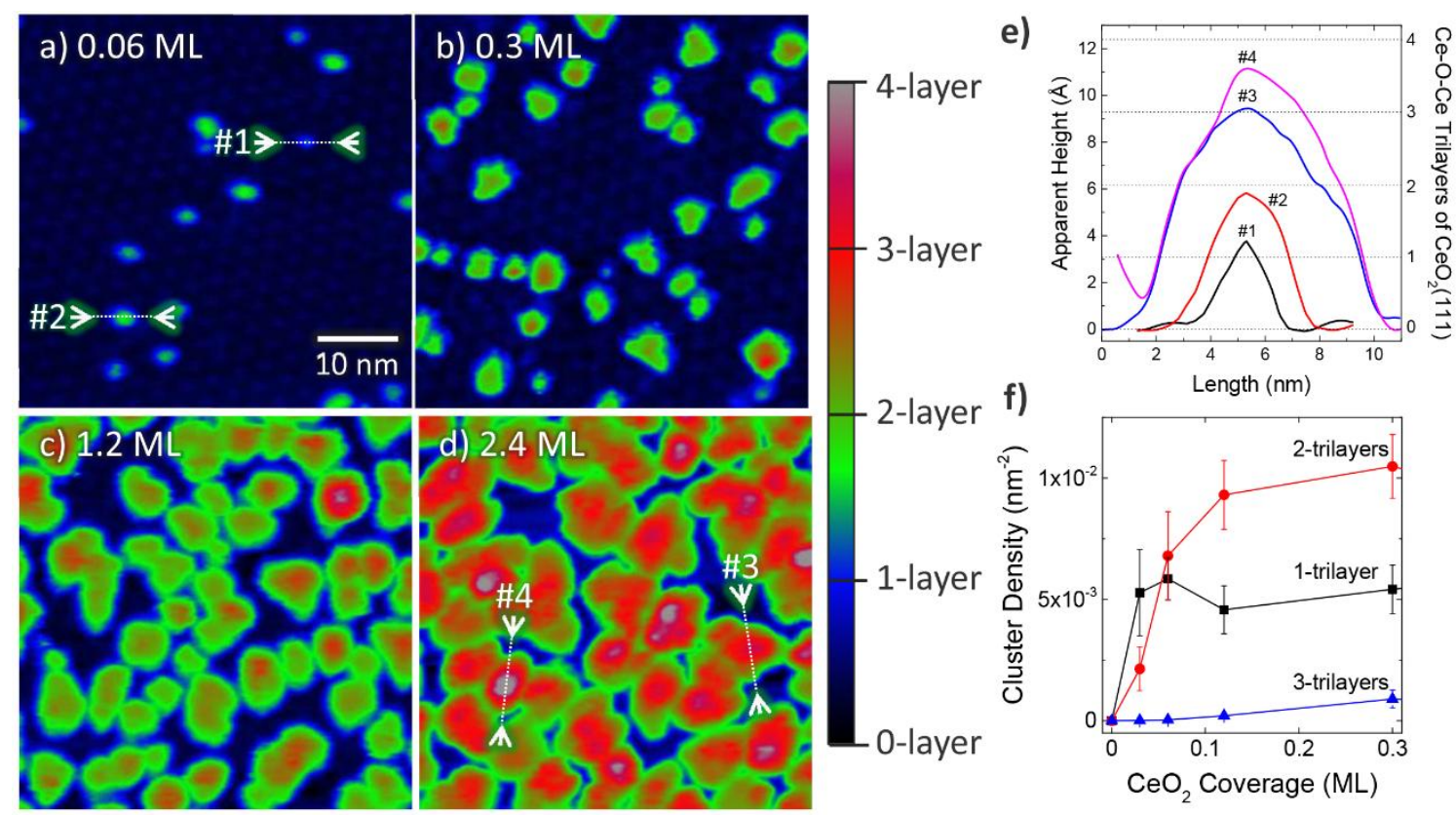

Fig. 4: (Color online) (a-d) Small-scale STM images showing the $\mathrm{CeO}_{x}$ cluster-height evolution with increasing coverage of Ce deposited at $300 \mathrm{~K}$ in $1 \times 10^{-7}$ Torr of $\mathrm{O}_{2}$. The color scale on the right shows the corresponding $\mathrm{CeO}_{2}(111)$-type $\mathrm{Ce}-\mathrm{O}-\mathrm{Ce}$ trilayer height. Height profiles of four $\mathrm{CeO}_{x}$ clusters (indicated in panels (a) and (d)) are shown in panel (e). Panel (f) shows the evolution of cluster heights for very low coverages of $\mathrm{CeO}_{x}(0-0.3 \mathrm{ML})$. Imaging conditions: $50 \times 50 \mathrm{~nm}^{2}, \mathrm{~V}_{\mathrm{s}}=-1.6 \mathrm{~V}, \mathrm{I}_{\mathrm{t}}=100 \mathrm{pA}$.

The size evolution of ceria clusters, deposited at 300K, is analyzed in Fig. 4. The STM images (a-d) are color coded to emphasize the different height levels. The STM line scans of Fig. 4(e), taken along the dotted lines in the STM images $(a, d)$, reveal a range of different height levels, showing a separation by an approximately constant level height of $\sim 3 \AA$, although clusters with a non-integer level height are also regularly observed (line profiles \#1, \#2 and \#4 in Fig. 4(e) deviate from the ideal $\mathrm{CeO}_{2}(111)$-type Ce-O-Ce trilayer height of $3.1 \AA$, later 
called layer). For small coverage of $\mathrm{CeO}_{x}(0-0.3 \mathrm{ML})$, the clusters are isolated and most of the clusters have height in the 1-2 layer range, although for $0.3 \mathrm{ML}$, a small number of $\sim 3$-layer high clusters is also observed (see Fig. $4(a, b, f))$. The onset of cluster coalescence is observed at $0.6 \mathrm{ML}$, and becomes more pronounced at even higher coverage of $1.2 \mathrm{ML}$ (Fig. 4(c)) and 2.4 ML (Fig. 4(d)). At 1.2 ML, the vast majority of clusters have height between 2- and 3layers (see color coding in Fig. 4(c)), and for $2.4 \mathrm{ML}$ (Fig. 4d)), the average height of clusters is 〜-layers, although many clusters with height in the 2-4-layer range are regularly observed. We note that since the nucleation site of the $\mathrm{CeO}_{x}$ clusters is ascribed to defects in $\mathrm{Gr}$ film, the cluster heights shown in Fig. 4 represents the prototypical scenario, although higher/lower clusters can be observed in defect deficient/rich Gr areas. As indicated by our calibrated $\mathrm{O} / \mathrm{Ce}$ Auger peak ratio measurements and by ample evidence in the literature [2, $15,22,26,27]$, the ceria clusters prepared under the present reactive evaporation conditions are of $\mathrm{CeO}_{2}$ stoichiometry or very close to it. Although no clear influence of the growth temperature on the stoichiometry of thick $\mathrm{CeO}_{x}$ films was observed (see Section 2: Experimental), it will be shown below that a better crystallographic order of the $\mathrm{CeO}_{\mathrm{x}}$ clusters can be achieved at a higher growth temperature of $500 \mathrm{~K}$. In contrast, the shape of $\mathrm{CeO}_{x}$ clusters grown at $300 \mathrm{~K}$ does not exhibit any shape regularity even for high coverages of 2.4 ML (see Fig. 2(g) and Fig. 4(d), respectively), pointing to a rather poor structural order of these clusters.

\section{$\underline{3.2 \text { Ceria cluster growth at } 500 \mathrm{~K}}$}




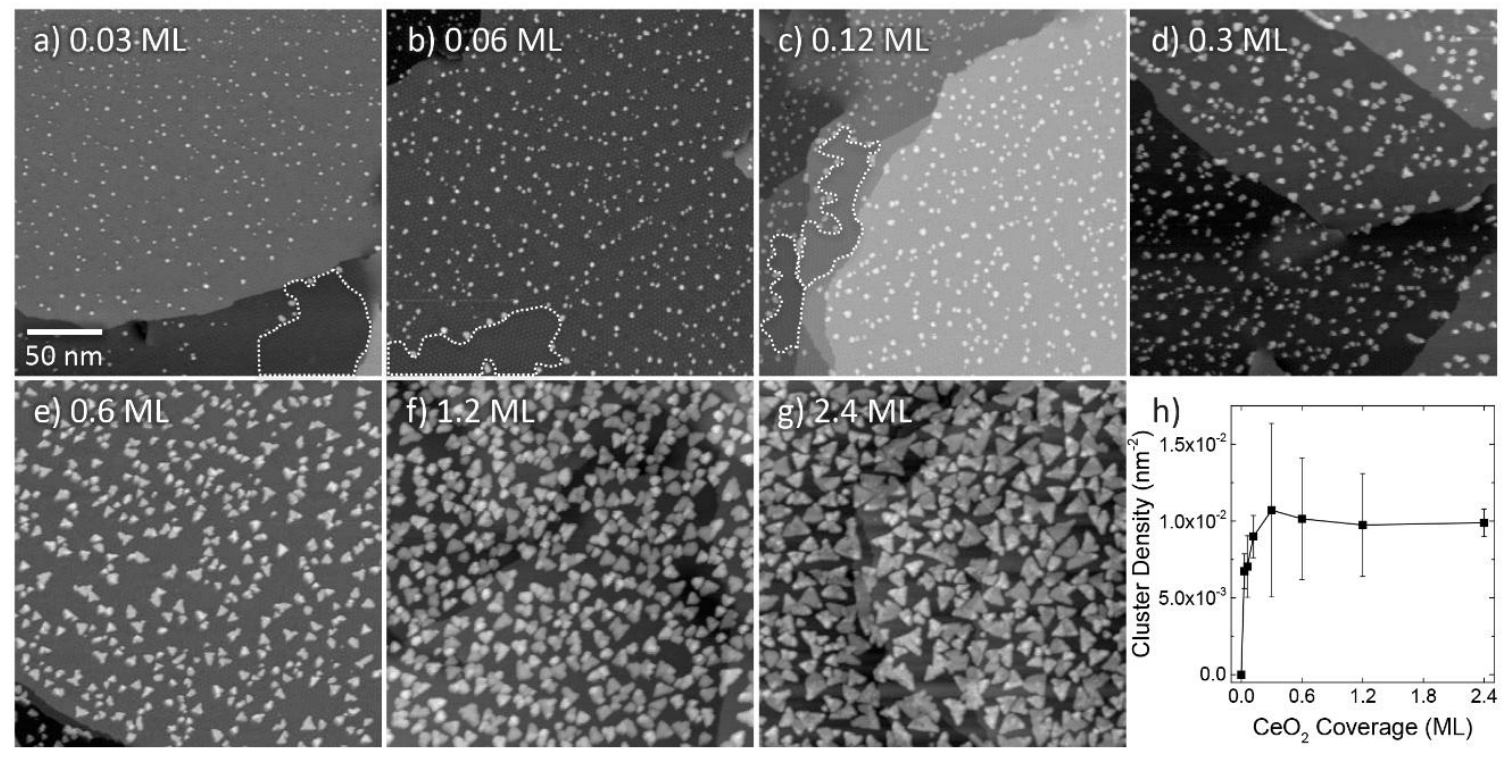

Fig. 5: Ceria nanoclusters deposited on $\mathrm{Gr}$ on $\mathrm{Ru}(0001)$ via reactive deposition of $\mathrm{Ce}$ in $1 \times 10^{-7}$ Torr of $\mathrm{O}_{2}$ at $500 \mathrm{~K}$. Panels (a-g) show STM images of $\mathrm{CeO}_{\mathrm{x}}$ nanoclusters with coverage increasing from $0.03 \mathrm{ML}$ to $2.4 \mathrm{ML}$. Correspondingly to Fig. 2, defectfree $\mathrm{Gr}$ Moiré areas, with no $\mathrm{CeO}_{x}$ clusters present, are highlighted by dotted lines in panels (a-c). Panel (h) shows the total density of clusters as a function of $\mathrm{CeO}_{\mathrm{x}}$ coverage, with error bars estimated from at least five different areas on the sample. Imaging conditions: $250 \times 250 \mathrm{~nm}^{2}, \mathrm{~V}_{\mathrm{s}}=-1.6 \mathrm{~V}, \mathrm{I}_{\mathrm{t}}=100 \mathrm{pA}$.

Ceria clusters grown by reactive deposition of cerium in $\mathrm{O}_{2}$ background at $500 \mathrm{~K}$ are investigated in Fig. 5. This growth temperature is the highest one that can be used without perturbing the graphene surface by intercalation of oxygen in between the graphene layer and the Ru surface [33]. At low ceria coverages, the cluster pattern is similar as seen for the growth at room temperature - see STM images 5(a-c). However, the total number of clusters is significantly reduced, by a factor of $\sim 2$, as evident from the cluster density plot in Fig. $5(\mathrm{~h})$. The cluster distribution is also non-uniform, and cluster-free surface areas can be recognized, in accordance with the proposed defect-mediated cluster growth mechanism (see areas encompassed with white dotted lines in Fig. $5(\mathrm{a}-\mathrm{c})$ ). The cluster number density 
(Fig. 5(h)) shows a steep increase at low coverages as for the $300 \mathrm{~K}$ growth (see Fig. 2(h)), but then saturates at a ceria coverage of 0.3-0.6 ML. This is in contrast to the behavior at 300 $\mathrm{K}$, where the cluster density increased further with coverage exceeding $1.2 \mathrm{ML}$. It thus appears that the secondary cluster nucleation sites, which are proposed to account for the slow increase in cluster numbers in Fig. 2(h), provide insufficient bonding strength to nucleate clusters at the higher growth temperature of $500 \mathrm{~K}$. For ceria coverages $>0.3 \mathrm{ML}$, the clusters develop triangular shapes while growing in size (STM images Fig. 5(d-g)).
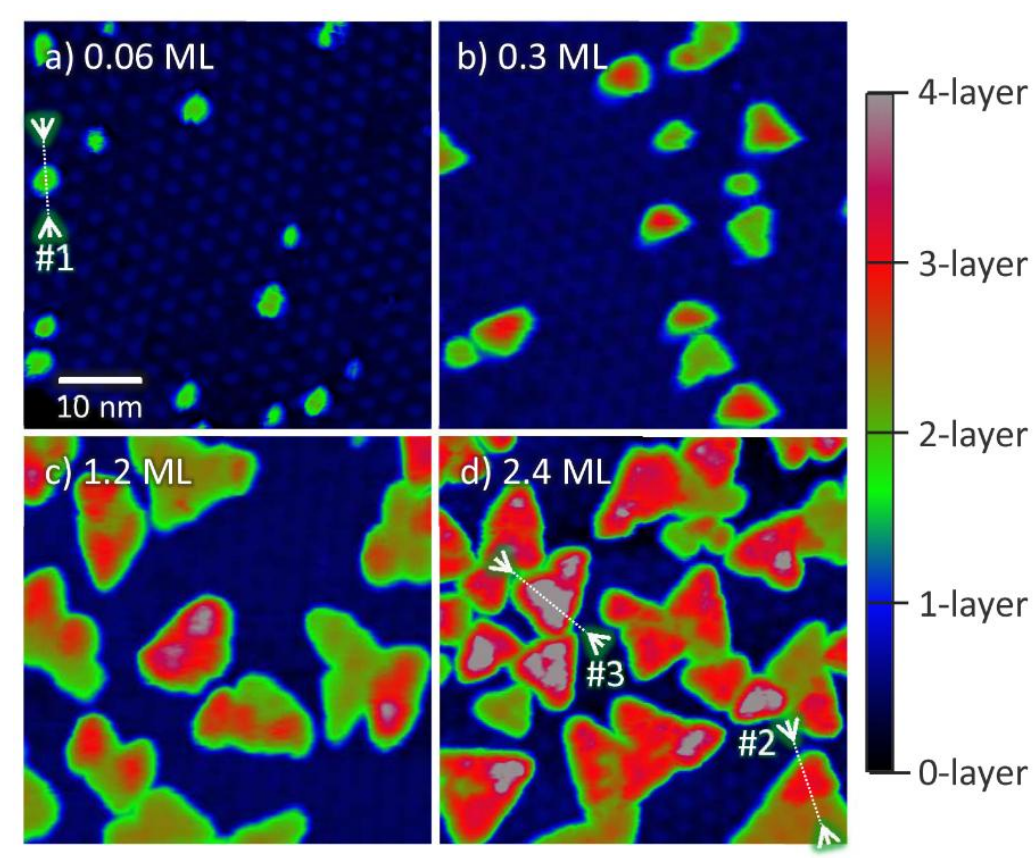

e)
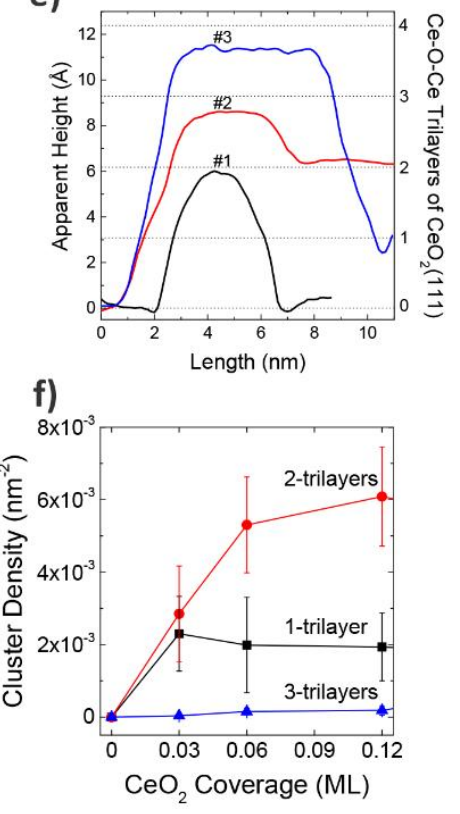

Fig. 6: (Color online) (a-d) Small-scale STM images showing the $\mathrm{CeO}_{\mathrm{x}}$ cluster-height evolution with increasing coverage of $\mathrm{Ce}$ deposited at $500 \mathrm{~K}$ in $1 \times 10^{-7}$ Torr of $\mathrm{O}_{2}$. The color scale on the right shows the corresponding $\mathrm{CeO}_{2}(111)$-type $\mathrm{Ce}-\mathrm{O}-\mathrm{Ce}$ trilayer height. At higher coverages (c-d), clusters preferentially adapt triangular shape, indicative of a better crystallinity compared to reactive Ce deposition at 300 K. Height line profiles for large coverages of $\mathrm{CeO}_{\mathrm{x}}$ show that the triangular clusters are flat, as shown in panel (e). Panel (f) shows the initial evolution of cluster heights 
for very low coverages of $\mathrm{CeO}_{x}(0-0.12 \mathrm{ML})$. Imaging conditions: $50 \times 50 \mathrm{~nm}^{2}, \mathrm{~V}_{\mathrm{s}}=-1.6$ $\mathrm{V}, \mathrm{I}_{\mathrm{t}}=100 \mathrm{pA}$.

STM images in Fig. 6 provide more information about the cluster shape and height. For the initial $\mathrm{CeO}_{x}$ coverages (0-0.12 ML), we observe small isolated clusters adapting mainly 1 - and 2-layer height (for $0.06 \mathrm{ML}$ shown in Fig. 6(a)), with only a very small number of clusters with the height corresponding to 3-layers (see Fig. 6(f)). This initial cluster height distribution is similar to growth at $300 \mathrm{~K}$ (compare Fig. 6(f) and Fig. 4(f)). At $0.3 \mathrm{ML}$ (Fig. 6(b)), the onset of coalescence is observed, and 3-layer high clusters are also regularly observed. 3-layer and 4layer clusters are observed at higher coverages (Fig. 6(c,d)), as also evident from the color coding of the STM images. Height line profiles for the larger coverage of $2.4 \mathrm{ML}$ show that these clusters are flat (see Fig. 6(e)). The areas of clusters with higher height increase on the surface after $500 \mathrm{~K}$ deposition, as compared to those at $300 \mathrm{~K}$, and the reduction of the number of clusters at $500 \mathrm{~K}$ is clearly apparent (compare $0.3 \mathrm{ML}$ or $1.2 \mathrm{ML}$ ceria deposited at $300 \mathrm{~K}$ and $500 \mathrm{~K}$ (Fig. 4(b,c) $(300 \mathrm{~K})$ ) versus Fig. 6(b,c) (500 K)). Interestingly, the line profiles taken at a large number of different coverages of $\mathrm{CeO}_{\mathrm{x}}$ grown at $500 \mathrm{~K}$ show a discrete height separation of $\sim 3 \AA$, compatible with the $\mathrm{CeO}_{2}$ (111)-like O-Ce-O trilayer structure element, although the layer spacing is contracted for second and higher layers - a consequence of a different density of states in the graphene and $\mathrm{CeO}_{2}$ - as reported previously on ceria thin films on metal substrates [14, 15, 27].

\section{$\underline{\text { 3.3 Annealing in UHV - The thermal stability of ceria nanoclusters }}$}



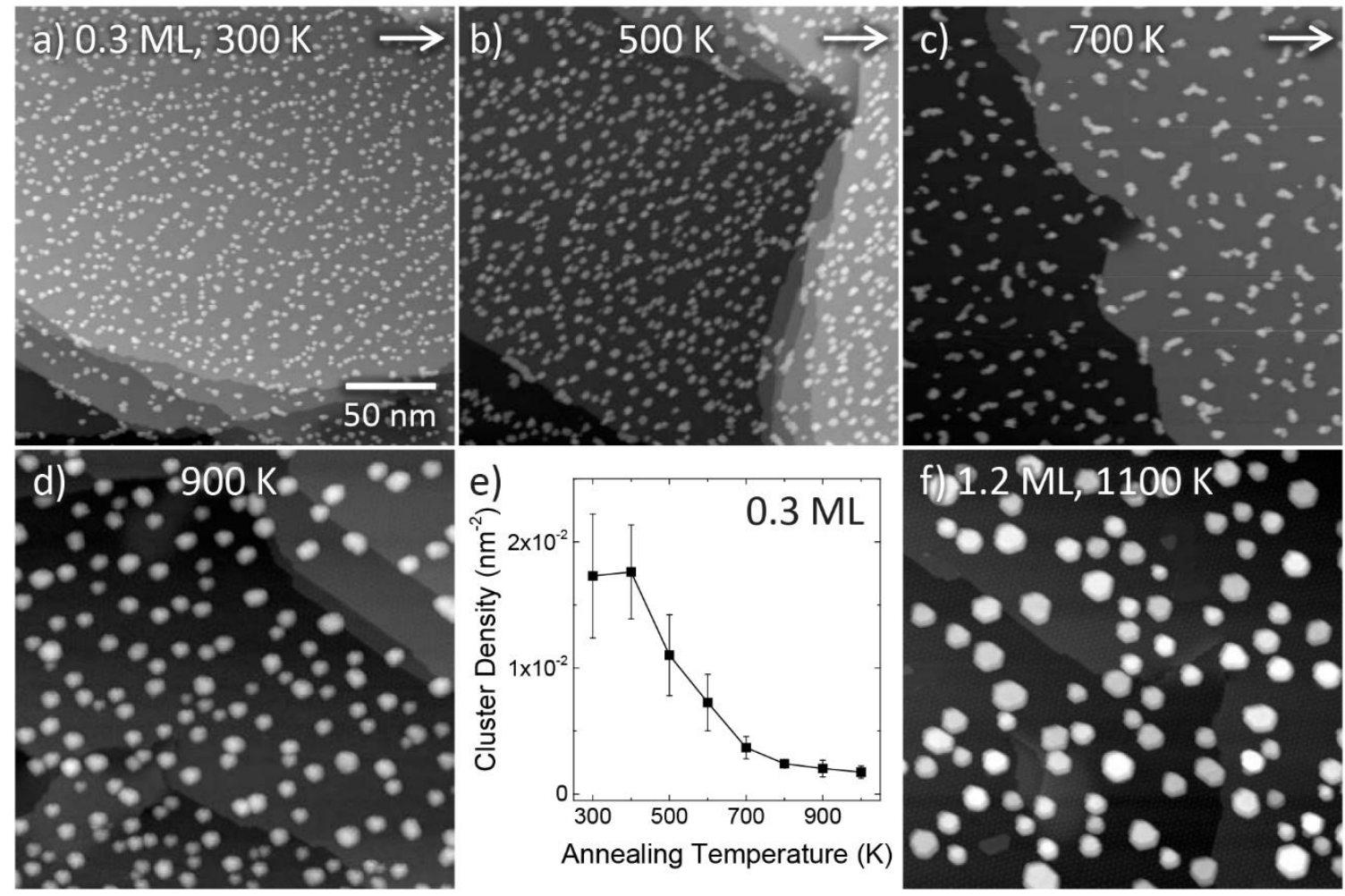

Fig. 7: Thermally-induced sintering of $\mathrm{CeO}_{x}$ nanoparticles. $0.3 \mathrm{ML}$ of Ce deposited in $1 \times 10^{-7}$ Torr $\mathrm{O}_{2}$ at $300 \mathrm{~K}(\mathrm{a})$ was heated in UHV. The annealing temperature was stepwise increased by $200 \mathrm{~K}$ increments and the sample was kept at the indicated temperature for 5 minutes before cooling down to $300 \mathrm{~K}$ and imaging. Images (a-d) show STM data for same $\mathrm{CeO}_{x}$ coverage of $0.3 \mathrm{ML}$ obtained within one experiment. The total density of clusters is shown in panel (e). In (f), data for the higher coverage of $1.2 \mathrm{ML}$ of $\mathrm{CeO}_{\mathrm{x}}$ are shown, demonstrating that after annealing to $1100 \mathrm{~K}, \mathrm{CeO}_{\mathrm{x}}$ clusters preferentially adapt hexagonal shapes. Imaging conditions: $250 \times 250 \mathrm{~nm}^{2}, \mathrm{~V}_{\mathrm{s}}$ $=-(1 \cdot 6-2.1) \mathrm{V}, \mathrm{I}_{\mathrm{t}}=25-100 \mathrm{pA}$

Fig. 7 displays the evolution of the clusters prepared by ceria deposition at $300 \mathrm{~K}$ (see Section 3.3) followed by the annealing in UHV, as seen by the STM for a ceria coverage of 0.3 ML (Fig. 7(a-d)). The cluster number remains stable up to $400 \mathrm{~K}$, but between $400-500 \mathrm{~K}$ cluster ripening sets in and the cluster density drops (Fig. 7(e)). After annealing at $700 \mathrm{~K}$ (Fig. 
7(c)), the clusters have changed their shape into elongated aggregates and their number density has decreased by a factor of $\sim 4$. The elongated shape of the aggregates is indicative of the mobility and attachment of the whole clusters via a Smoluchowski ripening mechanism [44]. After $900 \mathrm{~K}$ annealing, the clusters acquire more circular shapes and in some cases hexagonal structure elements can be discerned (Fig. 7(d)). This becomes more clearly apparent in Fig. 7(f), after annealing to $1100 \mathrm{~K}$; note that the latter STM image is from a surface with an initial ceria coverage of $1.2 \mathrm{ML}$. The hexagonal cluster shapes indicate that the ceria nanoparticles, although strongly reduced (see below), have restructured into $\mathrm{CeO}_{2}$ (111)-type crystallites during the high temperature treatment.

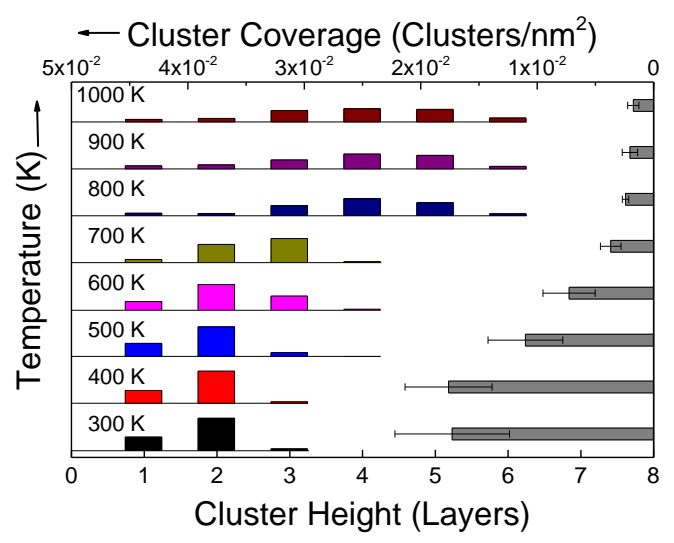

Fig. 8: (Color online) Cluster size distribution of $0.3 \mathrm{ML} \mathrm{CeO}^{-}-\mathrm{Gr} / \mathrm{Ru}(0001)$ during UHV annealing, obtained from images shown in Fig. 7. Top axis: Total number of clusters, as represented with grey bars on the right (different way of plotting data shown in Fig. 7(e)). Bottom axis: cluster height distribution, normalized with respect to the total number of clusters. One layer corresponds to the height of a $\mathrm{CeO}_{2}(111)$-type Ce-O-Ce trilayer.

The ripening of the clusters as expressed by the development of the cluster height distribution as a function of annealing temperature is plotted in Fig. 8 in the form of height 
histograms of normalized cluster numbers; the total numbers of clusters at the respective temperatures are included in the plot at the right hand side (bars corresponding to the axis on top). The analysis has been applied to the surface with $0.3 \mathrm{ML}$ ceria initial coverage that were deposited at $300 \mathrm{~K}$. The frequency of 1-layer and 2-layer clusters decrease slowly with increasing temperature up to $600 \mathrm{~K}$, at which temperature the ripening process has created a significant number of 3-layer clusters. The distribution of 3-layer clusters peaks at $700 \mathrm{~K}$ and then their number decreases again, and 4- and 5-layer clusters become dominant at $\mathrm{T} \geq$ $800 \mathrm{~K}$, with a small number of 6-layer clusters eventually created. This cluster distribution with annealing temperature, i.e. the shift to higher cluster sizes and the concomitant loss in the number density of clusters, is compatible with a Smoluchowski ripening mechanism, i.e. the migration and aggregation of the entire ceria clusters [44].

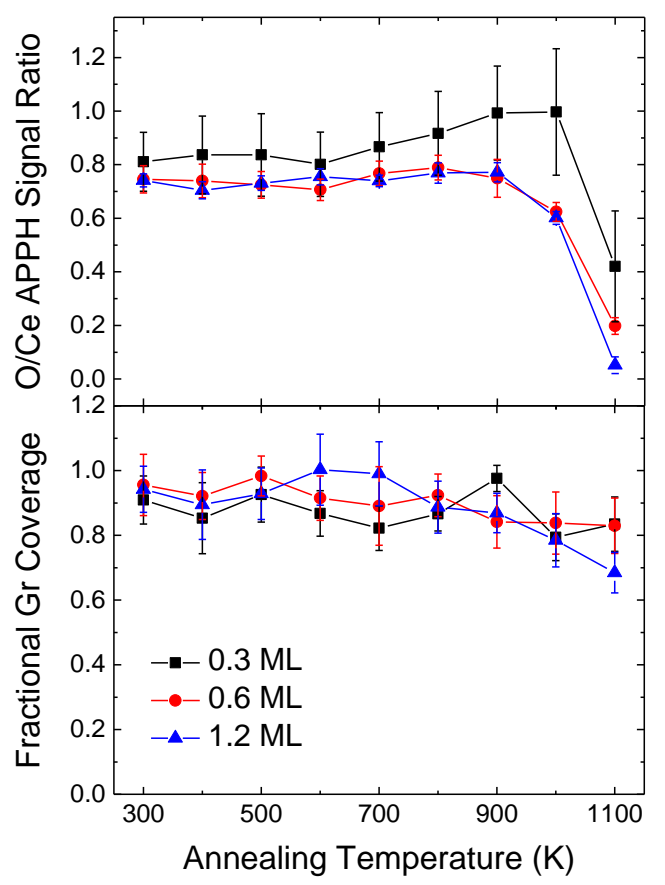

Fig. 9: (Color online) Thermal stability of nanoclusters during UHV annealing for three different $\mathrm{CeO}_{x}$ coverages: 0.3 ML (black squares), $0.6 \mathrm{ML}$ (red circles) and 1.2 
$\mathrm{ML}$ (blue triangles). Upper panel shows the ratio of $\mathrm{O} \mathrm{KL}_{23} \mathrm{~L}_{23} / \mathrm{Ce} \mathrm{N}_{45} \mathrm{O}_{23} \mathrm{~N}_{67} / \mathrm{V}$ Auger peaks, lower panel renders the fractional Graphene coverage.

The thermal stability of ceria nanoclusters has been investigated by Auger electron spectroscopy: the ratio of the $O \mathrm{KL}_{23} \mathrm{~L}_{23}$ (at $\sim 510 \mathrm{eV}$ ) and the $\mathrm{Ce}_{\mathrm{N}_{45}} \mathrm{O}_{23} \mathrm{~N}_{67} / \mathrm{V}$ Auger peaks has been recorded as a function of the annealing temperature for three different ceria coverages $(0.3,0.6,1.2 \mathrm{ML})$. Fig. 9 shows the ratios of the $\mathrm{O} / \mathrm{Ce}$ peak heights as a function of annealing temperature (upper panel). The ratio stays constant for all ceria coverages up to at least 900 $\mathrm{K}$ annealing in UHV, indicating a remarkable stability of the ceria nanoclusters on graphene against reduction. At $1100 \mathrm{~K}$, the loss of oxygen signals the decomposition of the ceria particles. The lower panel of Fig. 9 gives the fractional graphene coverage, as evaluated by AES (see section 2: Experimental). The graphene is a stable support layer for the ceria nanoclusters up to $900-1000 \mathrm{~K}$; a slight decrease of the graphene carbon AES signal for $\mathrm{T} \geq$ $1000 \mathrm{~K}$ indicates that upon ceria decomposition a small part of the graphene becomes etched away, possibly resulting in formation of $\mathrm{CO} / \mathrm{CO}_{2}$ gas molecules. Additionally, the thermal stability of ceria clusters up to $900 \mathrm{~K}$ supports the assignment of the sintering mechanism to Smoluchowski ripening, i.e. the migration and aggregation of the entire ceria clusters [44].

The stability of the ceria nanoclusters on graphene/Ru against reduction upon vacuum annealing is of mention in view of the easy reducibility of ceria in general, and the reported enhancement of reduction of ceria ultrathin films or nanoparticles in contact with metal surfaces $[15,16,22,26,32]$. Indeed, theoretical calculations have predicted a dramatic reduction of the oxygen vacancy formation energy, and therewith a more facile reduction, in ceria nanoparticles [45] as compared to larger bulk-type structures. The present results 
demonstrate the chemically inert nature of the single layer of graphene on $R u(0001)$ in connection with oxide nanoparticle growth and highlight its potential as an excellent stable support for such systems.

\section{Conclusions}

The growth of ceria nanoclusters on a single layer of graphene on $\mathrm{Ru}(0001)$ has been investigated by scanning tunneling microscopy and Auger electron spectroscopy as a function of ceria coverage and growth temperature. The cluster distribution has been analyzed from STM images and a defect-mediated growth mechanism, with the intrinsic defects of the graphene layer acting as cluster anchoring centers, is proposed. The ripening of the ceria nanoclusters sets in at $400-500 \mathrm{~K}$, and a Smoluchowksi ripening mechanism is suggested on the basis of the evolution of the cluster distribution as a function of temperature. The ceria nanoclusters are robust against thermal reduction in ultrahigh vacuum up to high temperatures (900K), and so is the graphene support surface. It appears thus that the ceria-on-graphene/Ru(0001) surface is a promising system to be tested in model catalysis studies.

\section{Acknowledgements}

This work was supported by the US Department of Energy, Office of Basic Energy Sciences, Division of Chemical Sciences, Geosciences \& Biosciences and performed in EMSL, a national scientific user facility sponsored by the Department of Energy's Office of Biological and Environmental Research and located at Pacific Northwest National Laboratory (PNNL). PNNL 
is a multiprogram national laboratory operated for DOE by Battelle. FPN acknowledges the award of an Alternate Sponsored Fellowship at PNNL and financial support of the University of Graz.

\section{Funding Sources}

US Department of Energy, Office of Basic Energy Sciences, Division of Chemical Sciences, Geosciences \& Biosciences 


\section{References:}

[1] A. Trovarelli, P. Fornasiero, Imperial College Press, London, 2013.

[2] D.R. Mullins, Surf. Sci. Rep. 70 (2015) 42.

[3] H.L. Tuller, S.J. Litzelman, W. Jung, PCCP 11 (2009) 3023.

[4] I. Celardo, J.Z. Pedersen, E. Traversa, L. Ghibelli, Nanoscale 3 (2011) 1411.

[5] M.V. Twigg, Catal. Today 163 (2011) 33.

[6] W. Deng, M. Flytzani-Stephanopoulos, Angew. Chem. Int. Ed. 45 (2006) 2285.

[7] J.A. Rodriguez, S. Ma, P. Liu, J. Hrbek, J. Evans, M. Pérez, Science 318 (2007) 1757.

[8] C.T. Campbell, C.H.F. Peden, Science 309 (2005) 713.

[9] E. Aneggi, D. Wiater, C. de Leitenburg, J. Llorca, A. Trovarelli, ACS Catal. 4 (2014) 172.

[10] A. Satsuma, M. Yanagihara, K. Osaki, Y. Saeki, H. Liu, Y. Yamamoto, S. Arai, J. Ohyama, RSC Advances 4 (2014) 54187.

[11] J.B. Park, J. Graciani, J. Evans, D. Stacchiola, S.G. Ma, P. Liu, A. Nambu, J.F. Sanz, J. Hrbek, J.A. Rodriguez, Proc. Natl. Acad. Sci. USA 106 (2009) 4975.

[12] H.-J. Freund, G. Pacchioni, Chem. Soc. Rev. 37 (2008) 2224.

[13] D.R. Mullins, P.V. Radulovic, S.H. Overbury, Surf. Sci. 429 (1999) 186.

[14] J.L. Lu, H.J. Gao, S. Shaikhutdinov, H.J. Freund, Surf. Sci. 600 (2006) 5004.

[15] S. Eck, C. Castellarin-Cudia, S. Surnev, M.G. Ramsey, F.P. Netzer, Surf. Sci. 520 (2002) 173.

[16] C. Castellarin-Cudia, S. Surnev, G. Schneider, R. Podlucky, M.G. Ramsey, F.P. Netzer, Surf. Sci. 554 (2004) L120.

[17] E.L. Wilson, Q. Chen, W.A. Brown, G. Thornton, J. Phys. Chem. C 111 (2007) 14215.

[18] E. Napetschnig, M. Schmid, P. Varga, Surf. Sci. 556 (2004) 1.

[19] M. Alexandrou, R.M. Nix, Surf. Sci. 321 (1994) 47.

[20] V. Matolín, J. Libra, I. Matolínová, V. Nehasil, L. Sedláček, F. Šutara, Appl. Surf. Sci. 254 (2007) 153.

[21] T. Staudt, Y. Lykhach, L. Hammer, M.A. Schneider, V. Matolín, J. Libuda, Surf. Sci. 603 (2009) 3382.

[22] F. Dvořák, O. Stetsovych, M. Steger, E. Cherradi, I. Matolínová, N. Tsud, M. Škoda, T. Skála, J. Mysliveček, V. Matolín, J. Phys. Chem. C 115 (2011) 7496.

[23] W. Xiao, Q. Guo, E.G. Wang, Chem. Phys. Lett. 368 (2003) 527.

[24] U. Berner, K.-D. Schierbaum, Phys. Rev. B 65 (2002) 235404.

[25] D.C. Grinter, R. Ithnin, C.L. Pang, G. Thornton, J. Phys. Chem. C 114 (2010) 17036.

[26] P. Luches, F. Pagliuca, S. Valeri, PCCP 16 (2014) 18848.

[27] S.G. Ma, J. Rodriguez, J. Hrbek, Surf. Sci. 602 (2008) 3272.

[28] T. Ma, S. Surnev, F. Netzer, Materials 8 (2015) 5205.

[29] D.C. Grinter, C. Muryn, B. Santos, B.-J. Shaw, T.O. Menteş, A. Locatelli, G. Thornton, J. Phys. Chem. C 118 (2014) 19194.

[30] M. Cargnello, V.V.T. Doan-Nguyen, T.R. Gordon, R.E. Diaz, E.A. Stach, R.J. Gorte, P. Fornasiero, C.B. Murray, Science 341 (2013) 771.

[31] S. Surnev, A. Fortunelli, F.P. Netzer, Chem. Rev. 113 (2013) 4314.

[32] J.A. Rodríguez, J. Hrbek, Surf. Sci. 604 (2010) 241.

[33] Z. Novotny, F.P. Netzer, Z. Dohnálek, ACS Nano 9 (2015) 8617.

[34] C.A. Schneider, W.S. Rasband, K.W. Eliceiri, Nat. Methods 9 (2012) 671.

[35] J.I.J. Choi, W. Mayr-Schmölzer, F. Mittendorfer, J. Redinger, U. Diebold, M. Schmid, J. Phys.: Condens. Matter 26 (2014) 225003.

[36] W.Y. Xu, L. Huang, Y.D. Que, X. Lin, Y.L. Wang, S.X. Du, H.J. Gao, Chinese Physics B 23 (2014) 4.

[37] An incorrect diameter of QCM sensor ( 0.50 instead of $0.81 \mathrm{~mm}$ ) was used in Ref. [33]. This leads to a coverage correction factor of 0.38 . Additionally, $1 \mathrm{ML}$ was defined in Ref. [33] with respect to the $\mathrm{C}$ atom density in Graphene/Ru(0001) $=1.24 \times 10^{15}$ atoms $/ \mathrm{cm}^{2}$. Here, $1 \mathrm{ML}$ is defined relative to the 
density of Ce atoms of the $\mathrm{CeO}_{2}(111)$ surface $\left(7.88 \times 10^{14} \mathrm{Ce}\right.$ atoms $\left./ \mathrm{cm}^{2}\right)$. These two factors result in the overall conversion factor of 0.6 .

[38] D.W. Flaherty, Z. Dohnalek, A. Dohnalkova, B.W. Arey, D.E. McCready, N. Ponnusamy, C.B. Mullins, B.D. Kay, J. Phys. Chem. C 111 (2007) 4765.

[39] J.A.D. Matthew, G. Strasser, F.P. Netzer, J. Phys. C: Solid State Phys. 15 (1982) L1019.

[40] D.W. Goodman, J.M. White, Surf. Sci. 90 (1979) 201.

[41] M.J. van Staden, J.P. Roux, Appl. Surf. Sci. 44 (1990) 259.

[42] Y. Pan, M. Gao, L. Huang, F. Liu, H.J. Gao, Appl. Phys. Lett. 95 (2009) 093106.

[43] Z. Zhou, F. Gao, D.W. Goodman, Surf. Sci. 604 (2010) L31.

[44] Y. Fukamori, M. Konig, B. Yoon, B. Wang, F. Esch, U. Heiz, U. Landman, ChemCatChem 5 (2013) 3330.

[45] A. Migani, G.N. Vayssilov, S.T. Bromley, F. Illas, K.M. Neyman, J. Mater. Chem. 20 (2010) 10535. 